\title{
Anti-noise Performance and Parameter Estimation Accuracy of FFT and FT Discrete Spectrum Correction
}

\author{
Kang Ding, Wenying Zhu, Zhijian Yang and Weihua Li \\ 1 School of Mechanical \& Automotive Engineering, South China University of Technology, Guangzhou 510640,PR China \\ 2 Guangdong Key Laboratory of Automotive Engineering, Guangzhou 510640, PR China
}

\begin{abstract}
The parameters estimation accuracy of Continuous Zoom FFT Spectrum by Fourier Transform under noiseless and Gaussian white noise is investigated. The relationship between parameters estimation errors and zoom multiples is revealed in the presence of noise-free signal. The probability of finding wrong maximum spectral line is increased with the rise of zoom multiples when the signal is accompanied with Gaussian white noise. Considering the influence of frequency resolution on frequency estimation accuracy and the probability mentioned above, two new indicators are proposed to evaluate the estimation accuracy. Based on above analysises, optimal zoom multiples under different signal-to-noise ratios are presented and a method is proposed to improve accuracy of this method. The results of simulations demonstrate that the improved method has higher correction accuracy and better anti-noise performance.
\end{abstract}

Keywords-fourier transform; anti-noise performance; spectrum correction; parameter estimation

\section{INTRODUCTION}

At present, for single harmonic component and far spaced multi-frequency components in discrete spectrum, there are altogether four kinds of methods employed to correct amplitude spectrum or power spectrum:Ratio Correction of Amplitude Spectrum,Three Convolution Correction of Discrete Spectrum[1-2], Continuous Zoom FFT Spectrum by Fourier Transform (hereinafter referred to as "FFT and FT Method") [3] and Phase Difference Correction [4].These methods all have high parameter estimation accuracy when the signals haven't accompanied with noise. However, the observed signals are always accompanied with noise, which will affect the parameter estimation accuracy. Consequently, analysis of noise influence on estimation accuracy of all kinds of discrete spectrum correction methods are of great significance. Schoukens [5] and Offelli [6] have researched the performance of Ratio Correction of Amplitude Spectrum method under noise. Qi Guo-qing [7] has investigated the frequency estimation accuracy of sinusoid on the interpolated FFT. Analysis of noise influence on the performance of phasedifference method is investigated by Li-Min $\mathrm{Zhu}$ [8] under condition that shifting points equal to FFT points. The antinoise performance and improvement measures of both window-length changing phase difference correction method and time-shifting correction method of phase difference in the presence of Gaussian white noise are presented by Yang Zhi- jian and Ding Kang [9]. However, systematic analysis about parameters estimation accuracy of FFT and FT Spectrum Correction Method is not presented until now. The FFT and FT Method was proposed by Liu Jin-Ming in 1995 [3]. This method utilizes FFT to generate a full-spectrum firstly, then zooms interested frequency regions of FFT spectrum with continuous FT algorithm. Consequently, precise value of some main frequency can be obtained, and thus greatly improve the frequency discrimination of FFT spectrum. The algorithms of this method are introduced as follows.

Firstly, a special Fourier transform form is obtained by three steps (change integration form into summation form, change the continuous form of time-domain parameter into discrete form and cut time-domain sample sequence to be finite).

$$
\mathbf{X}(f)=\sum_{n=0}^{N-1} x(n) e^{-j 2 \pi n \frac{f}{f_{s}}} \quad n=0,1, \ldots, N-1
$$

Where $x(n)=A_{0} \cos \left(2 \pi f_{0}^{1} n / N+\theta_{0}\right)$ is a discrete sequence of single harmonic signal. $f_{0} 、 A_{0}$ and $\theta_{0}$ are frequency, amplitude and phase of the signal respectively. $f_{0}^{1}=f_{0} / \Delta f$ is normalized frequency, $\Delta f=f_{\mathrm{s}} / N$ is the interval of spectral lines (which is also called frequency resolution). $N$ is the number of FFT points, $f_{\mathrm{s}}$ is sampling frequency. Equation (1) shows that time-domain is discrete while frequency-domain is continuous which is different from the norm form of Fourier transform.

Secondly, a given frequency regions $\left[f_{1}, f_{2}\right]$ is zoomed, the zoom multiples is $D$, then the resolution ratio of frequency is

$$
\Delta f^{\prime}=\Delta f / D \text {. }
$$

Hence, the calculation order of frequency sequence can be written as

$$
\left\{f_{1}, f_{1}+\Delta f^{\prime}, f_{1}+2 \Delta f^{\prime}, \ldots, f_{2}\right\} .
$$

Finally, the amplitude spectrum and phase spectrum of the above frequency sequence are calculated by using (1). Then the estimated frequency of harmonic signals can be obtained by choosing the maximum amplitude point's corresponding frequency value among these zoom spectral lines. 


\section{ESTIMATION ACCURACY OF FFT AND FT CORRECTION METHOD UNDER NOISE}

\section{A. Analysis of the maximum frequency error}

Assuming $\left[f_{1}, f_{2}\right]$ is the frequency regions which needs to be zoomed, when the true frequency of signal $f_{0}$ coincides with one discrete spectral line, the frequency obtained is precise. When $f_{0}$ is located in the centre of two discrete spectral lines, the frequency then represented have the maximum error, that is

$$
f_{0}-f_{1}=(i+0.5) \Delta f^{\prime} .
$$

Where $i$ is spectral line number after zoom .In this case, spectral line $i$ and $i+1$ have the same maximum amplitude value(without considering the interference of positive and negative frequency of single harmonic signal ). Therefore, no matter which spectral line's corresponding frequency value is selected to be the estimated value, the frequency error would be maximum, that is

$$
\nabla f_{\max }= \pm 0.5 \Delta f^{\prime} \text {. }
$$

The corresponding normalized maximum frequency error is

$$
\nabla f_{\text {max }}^{1}= \pm 0.5 \Delta f^{\prime} / \Delta f= \pm 0.5 / D \text {. }
$$

Obviously, $\nabla f_{\text {max }}^{1}$ is only relevant to zoom multiples. Thus the relation curve that $\nabla f_{\text {max }}^{1}$ varies with $D$ can be obtained as shown in Fig. 1 (a).

\section{B. Analysis of the maximum phase error}

The window function used for spectrum analysis must be moved to right for $T / 2$ points, thus causing the results of spectrum analysis has a phase shift

$$
\psi=-\pi \nabla f^{1}
$$

Where $\nabla f^{1}$ is normalized frequency error,this phase shift is the phase error.The maximum phase error can be obtained as

$$
\nabla \psi_{\text {max }}^{1}=-\pi \nabla f_{\text {max }}^{1} .
$$

Substituting (6) into (8), yields

$$
\nabla \psi_{\text {max }}^{1}= \pm 0.5 \pi / D \text {. }
$$

Therefore, the normalized maximum phase error is also only relevant to zoom multiples $D$, and has nothing to do with window function. Before zoom, i.e. when $D=1$, the maximum phase error is between $-\pi / 2 \sim \pi / 2$. This illustrates why the phase information in spectrum analysis is useless if no correction is carried out. However, the maximum phase error decreases obviously with the rise of zoom multiples. The relation curve that $\nabla \psi_{\text {max }}^{1}$ varies with $D$ can be obtained as shown in Fig.1 (b).

\section{Analysis of the maximum amplitude error}

The estimated formula of unilateral amplitude adding windows is[10]

$$
\hat{A}_{0}=\frac{2 a}{N}\left|\mathbf{X}\left(\hat{f}_{0}\right)\right| \text {. }
$$

Where $a$ is amplitude recovery coefficient of adopted window. For Rectangular window, Hanning window and Hamming window, $a$ is $1 、 2$ and $1 / 0.54$ respectively [11]. $\hat{f}_{0}$ is the estimated value of frequency. Meanwhile, when adding symmetric window function whose length is $\mathrm{N}$ to a harmonic signal, its unilateral continuous amplitude spectrum formula is

$$
\left|\mathbf{X}\left(\hat{f}_{0}\right)\right|=\frac{N}{2} A_{0}\left|\mathbf{W}\left(\hat{f}_{0}-f_{0}\right)\right|=\frac{N}{2} A_{0}|\mathbf{W}(\nabla f)|
$$

Where $\mathbf{W}(f)=\sum_{n=0}^{N-1} w(n) e^{-j 2 \pi f \frac{n}{f_{s}}} \quad$ is spectrum window function, $\nabla f=\hat{f}_{0}-f$ is the frequency estimation error. When (11) is substituted into (10), the estimated formula of amplitude can be obtained as follow

$$
\hat{A}_{0}=a A_{0}|\mathbf{W}(\nabla f)| \text {. }
$$

Substituting the maximum frequency error which can be seen in (5) into(12), the maximum amplitude error rate is obtained.

$$
\begin{aligned}
\nabla A_{\max } & =\left|\frac{A_{0}-a A_{0}\left|\mathbf{W}\left(\nabla f_{\max }\right)\right|}{A_{0}}\right| \times 100 \% . \\
& =|1-a| \mathbf{W}\left(0.5 \Delta f^{\prime}\right) \mid \times 100 \%
\end{aligned}
$$

As $\Delta f^{\prime}$ is associated with $D$, it can be seen from (13) that the maximum amplitude error is not only related to window function, but also is associated with $D$. The relation curves that $\nabla A_{\max }$ varies with $D$ under different windows can be seen from Fig. 1 (c).

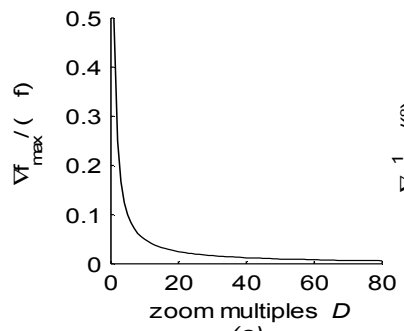

(a)

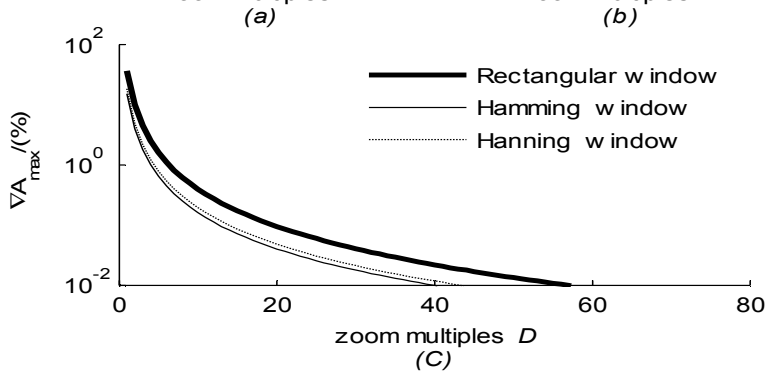

Figure1. Relation curves that maximum error in each parameter varies with zoom multiples 
Fig.1 shows that maximum errors decrease with increasing of $D$, it means estimation accuracy of frequency, phase and amplitude all increase with the rise of zoom multiples. The maximum error of each parameter is so little when zoom multiples is larger than 40 that can be neglected.

\section{ESTIMATION ACCURACY OF FFT AND FT CORRECTION METHOD UNDER WHITE GAUSSIAN NOISE}

The sequence from equal interval sampling to harmonic signals accompanied with additive white Gaussian noise can be written as

$$
s(n)=x(n)+z(n), \quad n=0,1, \ldots N-1 .
$$

Where $z(n)$ is the white Gaussian noise sequence, whose variance is $\sigma^{2} \cdot R \mathrm{sn}=A^{2} /\left(2 \sigma^{2}\right)$ is signal-to-noise ratios of sequence $s(n)$. In order to analyse the influence of Gaussian white noise on the parameter estimation accuracy of FFT and FT method, the spectrum of $s(n)$ is zoomed. Simulations are conducted for 10000 times to get the probability distribution of each spectrum line's amplitude to be the maximum. A harmonic signal $x(n)$ is produced by computer,whose amplitude is $A_{0}=1$, normalized frequency is $f_{0}^{1}=32$, phase is $\theta_{0}=24.5^{\circ}$. With sampling frequency $1024 \mathrm{~Hz}$, and carrying out FFT (1024 points), the frequency interval is $1 \mathrm{~Hz}$ while after zoom it changes into $A^{\prime}=1 / D$. Under different $R$ sn and $D$, the normalized frequency regions $[31,33]$ is zoomed using FFT and FT method. In the process of simulations, theory frequency spectral line is always located in the centre of two zoom spectral lines, so as to analyse maximum error. The results can be seen from Fig.2 and Fig. 3.

Fig. 2 shows that under same $R$ sn, the larger the zoom multiples is, the smaller the probability of finding the right maximum spectrum line is. As rectangular window narrower than Hanning window, the effect of adding rectangular window is better than that of adding Hanning window (However, in order to reduce interference among various frequencies, Hanning window is usually added in practical applications.).

Fig.3 shows that under the same zoom multiples, the smaller the $R \mathrm{sn}$ is, the smaller the probability of finding the right maximum spectral line is.

The above results show that if zoom multiples is too small, frequency estimation accuracy can not be guaranteed as frequency resolution is too large.If zoom multiples is too large, frequency estimation accuracy can not be further improved as the probability of finding the right maximum spectral line decreases simultaneously. It will only increase the calculated amount. Obviously, to ensure the frequency estimation accuracy and not waste calculated amount, a suitable zoom multiples under different circumstances should be chosen.
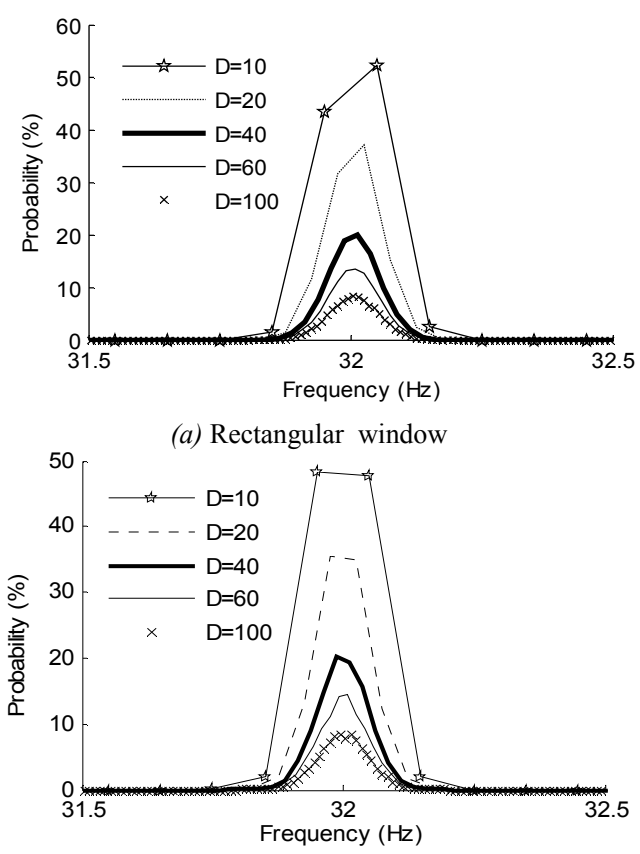

(b) Hanning window

Figure2. Probability distribution of each spectrum line's amplitude be the maximum $(\mathrm{Rsn}=0 \mathrm{~dB})$
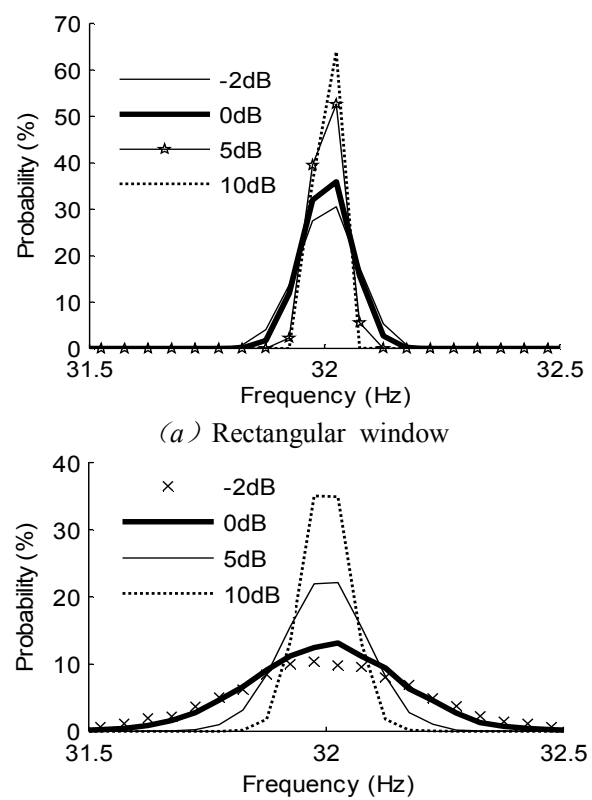

(b) Hanning window

Figure.3. Probability distribution of each spectrum line's amplitude be the maximum $(\mathrm{D}=20)$

In order to determine the appropriate zoom multiples, the indicator normalized frequency estimation comprehensive error (NFE for short) is proposed as formula (15). It can be used to evaluate the frequency estimation accuracy corresponding to different zoom multiples. The bigger the value of NFE is, the lower the estimation accuracy is. NFE can be written as 


$$
\text { enf }=\sum_{i=0}^{2 D} f_{1}^{1}+i\left|D-f_{0}^{1}\right| P(i)
$$

Where $f_{1}^{1}=f^{1} / \Delta f$ is normalized initial frequency, $P(i)$ is probability of the spectral line $i$ getting the maximum amplitude, $\left|f_{1}^{1}+i / D-f_{0}^{1}\right|$ stands for the deviation of spectral line $i$ relative to normalized theory frequency.

Another indicator normalized frequency estimation maximum error (NFEM for short) can be written as

$$
\operatorname{errf}_{\max }=\max \left(\left|f_{1}^{1}+i_{e} / D-f_{0}^{1}\right|\right) .
$$

Where $i_{e}$ stands for all the value $i$ which meets the following condition: $P(i)>0 \quad(i=0, \ldots, 2 D)$.

errf reflects the average frequency estimation error, while errf $f_{\max }$ reflects the credibility of the estimated results. The smaller the value of NFEM is, the higher the credibility of estimated results is. Through simulations, the relation curves that $\operatorname{errf}$ and $e r r f_{\max }$ varies with $D$ under different $R$ sn can be obtained as Fig. 4 .

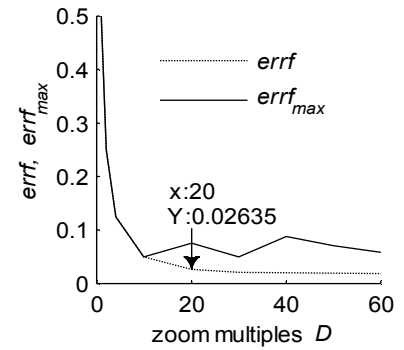

(a) Rectangular window $R \mathrm{sn}=-2 \mathrm{~dB}$

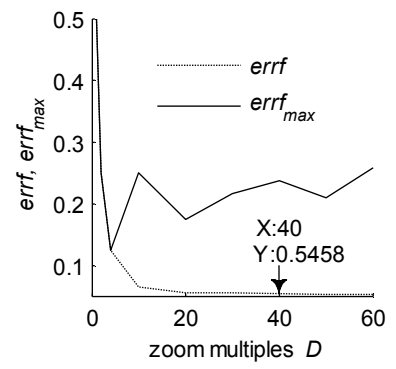

(c) Hanning window $R \mathrm{sn}=-2 \mathrm{~dB}$

Figure4.errf and $\operatorname{errf} f_{\text {max }}$ vary with $D$ under different $R$ sn

From Fig.4, the following conclusions can be drawn:

a) With the increasing of $D$, errf and errf $f_{\max }$ are reduced significantly at first then show a stable trend. It shows that, if $D$ is too small, frequency estimation accuracy will become too low. However, when $D$ increases to a certain extent, that is when the error is stable, it is useless for the improvement of accuracy if continue to increase $D$. b) With the increasing of $R \mathrm{sn}$, errf and $\operatorname{errf}_{\text {max }}$ both are reduced significantly. This means that noise has a great influence on frequency estimation accuracy. When $R \mathrm{sn}$ is high, larger zoom multiples can be selected to further improve estimation accuracy.

c) errf and errf max $_{\text {max }}$ match together at first then separate. After the turning point they both tend to be stable and maintain a relatively small value. That is to say, choosing the zoom multiples corresponding to turning point can not only guarantee a better estimation accuracy, but also guarantee a higher credibility, at the same time the calculate volume wasn't wasted. For Fig.4, the optimal zoom multiples can be selected separately as $D=10 、 D=40 、 D=4 、 D=18$. By using a similar method, Fig.5 which shows the optimal zoom multiples under different $R \mathrm{sn}$ is obtained.

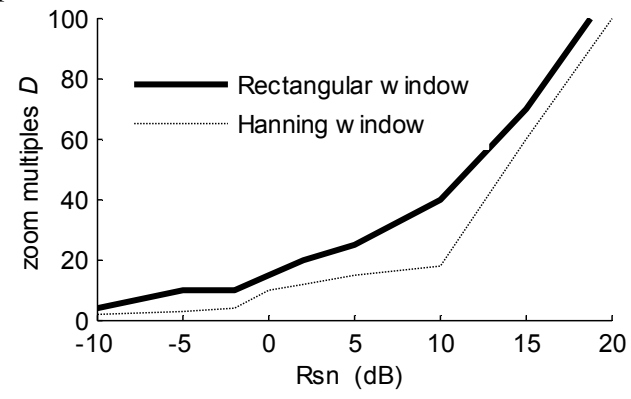

Figure5. The optimal $D$ under different $R$ sn

\section{IMPROVEMENT FOR FFT AND FT CORRECTION METHOD UNDER WHITE GAUSSIAN NOISE}

The foregoing analysis shows that noise has a great impact on the parameter estimation accuracy of FFT and FT correction. Considering that the decrease of estimation accuracy is because actual spectrum deviated from theory spectral lines, a nonlinear least square curve fitting method is proposed in order to reach a certain degree of de-noising effect. All the zoom spectrum lines are fitted to the curve form as shown in formula (11). This fitting process is the optimization problem

$$
\begin{aligned}
& \min F\left(\hat{A}_{0}, \hat{f}_{0}\right)=\left.\sum_{j=i \max -M}^{i \max +M}|| \mathbf{X}\left(f_{1}+j \Delta f^{\prime}-\hat{f}_{0}\right)|-| \mathbf{S}\left(f_{1}+j \Delta f^{\prime}\right)\right|^{2} . \\
& =\sum_{j=i \max -M}^{i \max +M}\left|\frac{N}{2} \hat{A}_{0}\right| \mathbf{W}\left(f_{1}+j \Delta f^{\prime}-\hat{f}_{0}\right)|-| \mathbf{S}\left(f_{1}+j \Delta f^{\prime}\right)||^{2}
\end{aligned}
$$

Where $\left|\mathbf{S}\left(f_{1}+j \Delta f^{\prime}\right)\right|$ is the amplitude of spectrum line $j$ of the signal $s(n), i \max$ stands for the value of $j$ when $\left|\mathbf{S}\left(f_{1}+j \Delta f^{\prime}\right)\right|$ is the largest. $M$ are the fitting data corresponding to spectrum lines which is located in the two sides of $i \max$. Then $\hat{A}_{0} 、 \hat{f}_{0}$ obtained through fitting can be the estimated values of amplitude and frequency respectively. The estimated value of phase is determined by the following formula 


$$
\hat{\theta}_{0}=\operatorname{angle}\left(\mathbf{S}\left(f_{1}+j \Delta f^{\prime}\right)\right)-\pi \hat{f}_{0} / \Delta f .
$$

Where angle $(\mathbf{S})$ stands for the phase of $\mathbf{S}$.

The following is an example to verify the feasibility of this approach. Add rectangular window to a harmonic signal firstly. The spectrum function of rectangular window is $W(f)=\sin (f)$. Then simulations are conducted for 1000 times (the parameters are seted completely identical with section 3 ) to estimate frequency using the improved method. The relation curves that errf and errf $f_{\max }$ vary with $D$ can also be obtained as shown in Fig.6. As can be seen from Fig.6, after improvement errf is reduced, the impact of noise is also much less than the original. For example, when $R \mathrm{sn}=10 \mathrm{~dB}$, the optimal zoom multiples can be selected separately as $D=50$ and $D=20$ when adding Rectangular window and Hanning window. This means the improved FFT and FT method has higher estimation accuracy and better anti-noise ability.
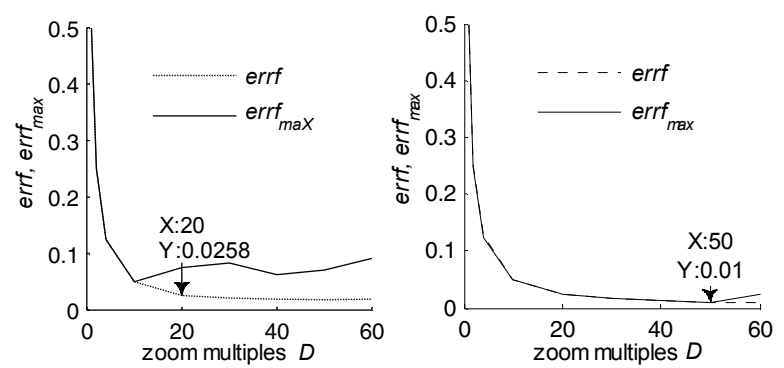

(a) Rectangular window $R \mathrm{sn}=-2 \mathrm{~dB}$

(b) Rectangular window $R \mathrm{sn}=10 \mathrm{~dB}$

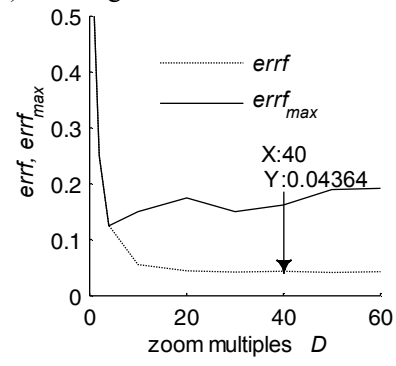

(c) Hanning window $R \mathrm{sn}=-2 \mathrm{~dB}$

Figure6. errf and $\operatorname{errf} f_{\mathrm{mx}}$ vary with $D$ under different $R$ sn (after improvement)

\section{CONCLUSIONS}

This article analyzed the parameter estimation error of the FFT and FT Spectrum Correction Method under the conditions of noiseless and Gaussian white noise. The estimation accuracy of each parameter (including frequency, phase and amplitude) has a great improvement with the increasing of $D$ in the absence of noise. The maximum error of each parameter can be neglected when zoom multiples is larger than 40. Under the influence of white Gaussian noise, the probability of finding right maximum spectrum line decreases with the increasing of $D$.To select the optimal zoom multiples, take the influence of frequency resolution on the frequency estimation accuracy and the probability of finding the wrong maximum spectrum line both into consideration, two evaluation indicators NFE and NFEM are proposed. The optimal zoom multiples under different $R$ sn are given finally.

At last, the FFT and FT spectrum correction method is modified and improved. We use nonlinear least square method to fit the largest spectral line and its around lines to the form of single harmonic spectrum curve under noiseless, so as to improve the accuracy of parameter estimation. The result of simulations show that the improved FFT and FT Correction Method makes the parameter estimation error smaller.

\section{REFERENCES}

[1] Ding Kang , Zhang Xiao-fei, “ Theory development of spectrum correction", Journal of Vibration Engineering, 2000,13(1):14-22.(in chinese)

[2] Ding Kang, Xie,Ming. “ Error analysis of three-point convolution amplitude correcting method on discrete spectrum".Journal of Vibration Engineering, 1996,9(1): 92-98.(in chinese)

[3] Liu Jin-ming, Ying Huai-qiao,"Zoom FFT spectrum by Fourier transform", Journal of Vibration Engineering, vol.8,no.2,pp.162-166, Jun, 1995

[4] Ding Kang, Xie Ming, "Phase difference correction method for phase and frequency in spectral analysis",Mechanical System s and Signal Processing,2000, 14 (5) : 835-843.

[5] Schoukens J, Pintelon R and van Hamme H, "The interpolated fast transform: a comparative study", IEEE Trans. Instrum. Meas, vol.42,no.2,pp:226-232, 1992

[6] Carlo Offelli and Dario Petri, "The influence of windowing on the accuracy of multifrequency signal parameter estimation", IEE Transactions on Instrumentation and Measurement,1992,41(2): 256261.

[7] Qi Guo-qing, Jia Xin-le, "Accuracy Analysis of Frequency Estimation of Sinusoid Based on Interpolated FFT", Acta Electronica Sinica, vol.32,no.4,Apr, pp.625-629, 2004.

[8] Li-Min Zhu, Han-Xiong Li,H.Ding, You-Lun Xiong, "Noise influence on estimation of signal parameter from the phase difference of discrete fourier transeforms", Mechanical Systems and Signal Processing,2002, 16(6): 991-1004.

[9] Yang Zhi-jian, Ding Kang, "Accuracy analysis of frequency estimated by applying time-shifting correcting method of phase difference in presence of Gaussian white noise" ,Journal of Vibration Engineering, vol.20,no.3, pp.274-279, Jun,2007

[10] Ding Kang, Xie Ming, Yang Zhijian, The Theory and Technology of Discrete Spectrum Correction, Beijng: Science Press, 2008, pp.111-119, pp.169-184.

[11] Xie Ming and Ding Kang, "Corrections for frequency, amplitude,and phase in a fast Fourier transform of a harmonic signal", Mechanical Systems and Signal Processing, 1996, 10(2), 211-221. 